Original Paper http://ajol.info/index.php/ijbcs http://indexmedicus.afro.who.int

\title{
Influence des haies de Jatropha sur le rendement d'une culture de sorgho (Sorghum vulgare) dans la zone Ouest du Burkina Faso: cas du terroir de Torokoro
}

\author{
Pascal BAZONGO ${ }^{1,2^{*}}$, Karim TRAORE ${ }^{1}$,Ouola TRAORE ${ }^{1}$, \\ Barthelemy YELEMOU ${ }^{4}$, Kadidia B. SANON ${ }^{3}$, Salifou KABORE ${ }^{1}$, Victor HIEN ${ }^{5}$ et \\ Bismarck H. NACRO ${ }^{2}$
}

\footnotetext{
${ }^{1}$ Institut de l'Environnement et de Recherches Agricoles (INERA), Département Gestion des Ressources Naturelles et Système de Production, INERA-Farako-Ba, Laboratoire Sol Eau Plante, BP 910 Bobo-Dioulasso, Burkina Faso.

${ }^{2}$ Université Polytechnique de Bobo, Ecole Doctorale, Sciences Naturelles et Agronomie 01 BP. 1091 BoboDioulasso 01, Burkina Faso.

${ }^{3}$ Laboratoire de Microbiologie, INERA/DPF, 03 BP 7047 Ouagadougou 03, Burkina Faso.

${ }^{4}$ Institut de l'Environnement et de Recherches Agricoles (INERA), Département Gestion des Ressources Naturelles et Système de Production, INERA-Saria,BP 10 Koudougou, Burkina Faso.

${ }^{5}$ Institut de l'Environnement et de Recherches Agricoles (INERA), Département Gestion des Ressources Naturelles et Système de Production, INERA-Kamboinsé, Laboratoire Sol Eau Plante, 01 BP 476 Ouagadougou 01, Burkina Faso.

*Corresponding author, E-mail:bazpasco@yahoo.fr;01 BP. 1091 Bobo-Dioulasso 01 Burkina Faso.
}

\section{REMERCIEMENTS}

Les auteurs traduisent leur gratitude à l'Union Européenne à travers le "10 ${ }^{e}$ Fond Européen de Développement". Les remerciements vont à l'endroit du programme de subvention de recherche de l'Union Africaine [HRST/ST/AURG/CALL1/2011], pour avoir financé cette étude. Les auteurs remercient également le Programme de Productivité Agricole en Afrique de l'Ouest (PAAAO) / WAAPP pour avoir supporté le financement de cette étude.

\section{RESUME}

Dans un contexte de désertification et avec la crise énergétique, un bon nombre d'acteurs de développement au Burkina Faso s'est intéressé au Jatropha curcas L. comme source d'énergie et de durabilité des productions. Afin d'évaluer l'influence de Jatropha sur les propriétés chimiques du sol et la productivité du sorgho associée, des essais ont été suivis pendant trois ans dans le terroir de Torokoro en zone Sud soudanienne de pluviométrie d'environ $1200 \mathrm{~mm}$. Les plants de Jatropha, âgés de 6 ans, sont plantés aux écartements de 5 $\mathrm{m}$ entre les lignes et $2 \mathrm{~m}$ entre les plants. Le dispositif expérimental comporte trois blocs composés chacun de deux parcelles de $400 \mathrm{~m}^{2}$ correspondants aux 2 traitements: une par celle avec l'association sorgho-Jatropha et une parcelle en culture pure de sorgho (témoin). Les prélèvements de sol ont été effectués sous houppier, à $1 \mathrm{~m}$ et $2 \mathrm{~m}$ de la haie. Les résultats indiquent que Jatropha améliore la teneur du sol en carbone de $10 \%$ et de $8 \%$ en matière organique par rapport au témoin. Jatropha est aussi source de phosphore et de potassium et influence positivement la productivité du sorgho. Jatropha améliore donc les propriétés chimiques du sol et la productivité du sorgho.

(C) 2015 International Formulae Group. All rights reserved.

Mots clés : Jatropha curcas L., sol, Sorgho, Rendements, propriétés chimiques, Burkina Faso. 


\title{
Influence of Jatropha hedges on the yield of a sorghum crop (Sorghum vulgare) in the western region of Burkina Faso: case of Torokoro locality
}

\begin{abstract}
In a context of desertification and with the energy crisis, a number of development actors in Burkina Faso became interested in Jatropha curcas L. as a source of energy and sustainability of production. To assess the influence of Jatropha on soil chemical properties and on the productivity of associated sorghum, experiments were followed for three years in the village of Torokoro in south sudanian region, with a rainfall of about $1200 \mathrm{~mm}$. Jatropha plants, aged 6 , are planted at $5 \mathrm{~m}$ between rows and $2 \mathrm{~m}$ between plants. The experiment consisted of three blocks each consisting of two plots of $400 \mathrm{~m}^{2}$ corresponding to two treatments: a plot with sorghum and Jatropha in association and a plot with sorghum only (control). Soil samples were taken under crown, at $1 \mathrm{~m}$ and $2 \mathrm{~m}$ from the hedge. The results indicate that Jatropha improves the carbon content in the soil of $10 \%$, and the organic matter content of $8 \%$ compared to the control. Jatropha is also a source of phosphorus and potassium and positively influences the productivity of sorghum. Therefore, Jatropha improves the chemical properties of soil and productivity of sorghum.
\end{abstract}

(C) 2015 International Formulae Group. All rights reserved.

Keywords: Jatropha curcas L., soil, Sorghum, yields, chemical properties, Burkina Faso.

\section{INTRODUCTION}

Les changements climatiques demeurent l'une des menaces les plus graves qui pèsent sur le développement durable (PANA, 2006). Au Burkina Faso, les manifestations les plus visibles des changements climatiques se rapportent à la sécheresse qui accélère le déboisement. C'est dans ce contexte de dégradation du couvert végétal que certains acteurs du monde rural se sont intéressés à des espèces d'arbres à usages multiples. Jatropha est apparu comme l'arbre idéal pour répondre à cette préoccupation. Il pourrait permettre la récupération et la protection des sols contre la dégradation et l'amélioration du niveau de la sécurité alimentaire. L'intérêt grandissant pour cette plante s'explique par sa capacité à pousser sur des terres marginales (Paramathma et al., 2007), à lutter contre l'érosion et à réhabiliter des terres peu fertiles (Henning, 2002; Pellet et Pellet, 2007; Blind et al., 2008; Domergue et Pirot, 2008). En outre, la culture de Jatrophacurcas L. semble améliorer la fertilité du sol (Diédhiou, 2009). Elle fait l'objet d'association de cultures vivrières en couloir sans compétition nuisible avec cellesci (Bazongo, 2011). La curcine de Jatrophaest une substance proche de la ricine qui a des propriétés insecticides (Adebowale et Adelire, 2006), fongicides (Ogbebor et al., 2007) et nématicides (Vedie et Geffroy, 2005). Cependant, depuis cette ferveur pour Jatropha au Burkina Faso, très peu d'études se sont intéressées à son impact sur l'environnement. Il existe très peu d'informations scientifiques sur les impacts de la culture de Jatropha sur les rendements des cultures et plus particulièrement du sorgho qui lui est souvent associé et sur les paramètres chimiques du sol. Les associations de cultures sorgho-Jatropha posent des inquiétudes car certains auteurs comme Low et Booth (2007) et Endelevu (2009) trouvent Jatropha envahissant. La possibilité que les producteurs adoptent cette culture au détriment des cultures vivrières a été évoquée par Lottman (2008), et Guillaume (2009) soutient le caractère envahissant de cette plante en évoquant un risque d'insécurité alimentaire avec son adoption par les agriculteurs. Par ailleurs, Jatropha produit des substances toxiques comme la curcine et l'acide cyanidrique (Henning et al., 2005) dont les effets sur les propriétés chimiques du sol, la croissance et le rendement de la culture de sorgho associé sont peu connus. C'est pour 
apporter des éléments de réponse à ces préoccupations que la présente étude a été conduite en vue d'évaluer l'effet d'une haie de Jatropha curcas L. sur la croissance et le rendement du sorgho.

\section{MATERIEL ET METHODES \\ Présentation du site d'étude}

Les travaux ont été conduits en milieu paysan dans la zone Sud-soudanienne du Burkina Faso, dans la localité de Torokoro de coordonnées $4^{\circ} 20^{\prime}$ longitude Ouest, de $9^{\circ} 59^{\prime}$ latitude Nord et $297 \mathrm{~m}$ d'altitude. La pluviométrie annuelle dans cette zone peut atteindre $1200 \mathrm{~mm}$ selon la Direction Provinciale de l'Agriculture, des Ressources Hydrauliques, de l'Assainissement et de la Sécurité Alimentaire (Tableau 1), avec une saison pluvieuse qui dure de 4 à 5 mois. Ce site a été choisi du fait des actions de vulgarisation antérieures de la culture de Jatropha curcas par certains promoteurs. Les sols du site de Torokoro sont de type ferrugineux tropical lessivé (lixisol) sur les versants. Quelques sols hydromorphes à pseudogley d'ensemble sont observés dans les interfluves tandis que les sommets de ceux-ci sont occupés par des lithosols (Youl, 2009).

\section{Matériel végétal}

Il est constitué de sorgho, variété Sariasso 1 ayant un cycle de 120 jours avec un potentiel de rendement de 3 tonnes/ha et des plants de Jatropha curcas L.

\section{Méthodologie}

\section{Choix des producteurs expérimentateurs}

Trois producteurs ont été choisis sur la base de leur capacité à respecter les itinéraires techniques des cultures mises en place et leur ouverture à accepter l'innovation.

\section{Dispositif expérimental}

L'essai a été conduit pendant 3 années, de 2012 à 2014 dans six (6) parcelles de champs de Jatropha de plants âgés de 6 ans. L'âge de 6 ans représente l'âge de la pleine production de Jatropha curcas. Le dispositif expérimental comporte trois blocs et chaque bloc est composé de deux parcelles correspondant aux 2 traitements suivants: une parcelle avec l'association de sorgho -Jatroph acurcas L., une en culture pure de sorgho servant de témoin. Chaque parcelle a une superficie de $400 \mathrm{~m}^{2}$ (20 m x $\left.20 \mathrm{~m}\right)$. Les blocs sont séparés de $5 \mathrm{~m}$ et les traitements, de 20 $\mathrm{m}$. Les parcelles en culture pure de sorgho servant de témoins ont été implantées sur une jachère de 10 ans. Jatropha curcas L. a été planté aux écartements de $5 \mathrm{~m}$ entre lignes et $2 \mathrm{~m}$ soit une densité de 1071 plants/ha. Le sorgho a été semé en couloir, dans l'espace se trouvant entre les lignes de Jatropha curcas L. soit $100 \mathrm{~m}^{2}(20 \mathrm{~m} \times 5 \mathrm{~m})$. Quatre (4) lignes de semis de sorgho ont été semées par couloir. Ainsi, dans le traitement correspondant à l'association sorgho-Jatropha, le sorgho occupe quatre (4) couloirs.

\section{Collecte des données \\ Prélèvement du sol}

Le prélèvement du sol a été effectué dans les six (6) parcelles et suivant 3 distances à savoir, D0 (sous houppier de Jatropha), àD1= $1 \mathrm{~m}$ du houppier, à $\mathrm{D} 2=2 \mathrm{~m}$ du houppier puis dans le sol témoin cultivé en sorgho pur. Pour chaque distance de prélèvement, trois échantillons de sol ont été prélevés sur l'horizon $0-20 \mathrm{~cm}$ puis un échantillon composite constitué. Au total, douze (12) échantillons ont fait l'objet d'analyse de laboratoire, soit 3 parcelles (association sorgho-Jatropha) x 3 distances de prélèvement +3 parcelles témoins $\mathrm{x} 1$ prélèvement.

\section{Analyses de laboratoire}

Les analyses ont concerné le pH (eau et $\mathrm{KCl})$, le phosphore total $(\mathrm{P})$, le phosphore assimilable, le carbone organique (C), l'azote total $(\mathrm{N})$ et le potassium total $(\mathrm{K})$. $\mathrm{Le}_{\mathrm{pH}}$ eau été mesuré à partir d'une solution de sol obtenu dans un rapport masse/volume de $(1 \mathrm{~g} /$ $2,5 \mathrm{ml})$. Le $\mathrm{pH}_{\mathrm{KCl}}$ a été déterminé à partir d'une solution de chlorure de potassium. Le P total a été mesuré sur le condensé de la minéralisation (BUNASOLS, 1987). Le phosphore assimilable a été déterminé par la 
méthode de BRAY 1 citée par Michaelson et al. (1987). L'azote total a été dosé par la méthode de Kjeldahl modifiée (Hillebrand et al., 1953). Le carbone organique a été déterminé selon la méthode de Walkley-Black (1934). Le taux de matière organique (MO) est déterminé suivant la formule: $\mathrm{MO}=$ Taux de carbone * 1,724. Le potassium total a été mesuré au spectrophotomètre à flamme à partir $\mathrm{du}$ reliquat du filtrat issu de la minéralisation des prises d'essai de sol.

\section{Paramètres mesurés}

Hauteur des plants de sorgho

Ces observations ont consisté à délimiter une superficie de $25 \mathrm{~m}^{2}$ dans chaque parcelle. Par parcelle, 10 plants soit au total 60 plants de sorgho ont été retenus pour déterminer la hauteur du sorgho à 30, 60 et 90 jours après semis. Les mesures ont été effectuées sur les mêmes plantes durant tout le cycle de la plante.

\section{Evaluation du rendement du sorgho}

Le poids total de panicules, le rendement paille et le rendement grains ont été déteminés à partir du carré de rendement de $25 \mathrm{~m}^{2}$.

\section{Analyses statistiques}

Les données collectées ont été traitées à l'aide du tableur Excel 2007 et soumises à une analyse de variance (ANOVA) à l'aide du logiciel XLSTAT-pro 7.5.2. Les moyennes des variables ont été comparées en utilisant le test de Newman-Keuls au seuil de probabilité $5 \%$.

\section{RESULTATS}

\section{Evaluation des paramètres chimiques du sol}

Les résultats d'analyses du Tableau 2 montre que le phosphore total présente des valeurs significativement différentes suivant les distances de prélèvement du sol $(\mathrm{p}=0,012)$. La teneur en phosphore total du sol est inversement proportionnelle à la distance de prélèvement du sol. On observe des augmentations de phosphore de $7 \%$ à $1 \mathrm{~m} \mathrm{du}$ houppier et de $16 \%$ sous houppier de Jatropha par rapport à la teneur du phosphore total dans le sol sans Jatropha. Des différences significatives $(\mathrm{P}<0,05)$ sont obtenues entre les traitements pour les paramètres mesurés comme les taux de matière organique (MO) et de phosphore assimilable ( $\mathrm{P} \_$assimilable). Les taux de matière organique et de phosphore assimilable augmentent sous houppier de Jatropha par rapport au sol témoin sans Jatropha. La teneur en matière organique augmente de $8 \%$ sous houppier de la haie de Jatropha par rapport au sol témoin sans Jatropha. La teneur en carbone des sols prélevés sous les plantations de Jatropha demeure plus élevée de $10 \%$ que celle du sol témoin sans Jatropha. On observe que la teneur en azote du sol témoin sans Jatropha est équivalente à celle du sol prélevé sous le houppier de Jatropha $(0,05 \pm 0,01)$.

\section{Hauteur des plants de sorgho}

Trente jours après les semis (30 JAS), on observe dans le Tableau 3 des différences significatives entre les traitements comparés pour la hauteur des plants de sorgho associé au Jatropha et ceux en culture pure $(\mathrm{P}<0,05)$, et ce, en première comme en deuxième année d'expérimentation. En troisième année et à soixante jours après semis (60 JAS), on observe des différences significatives $(\mathrm{P}<0,05)$ entre les plants de sorgho associés au Jatropha et ceux en culture pure. Pendant les trois années d'expérimentation, on observe à 90 jours après semis, des différences significatives $(\mathrm{P}<0,05)$ entre les hauteurs des plants de sorgho qu'ils soient associés au Jatropha et les plants de sorgho en culture pure.

\section{Nombre et poids de panicules de sorgho}

Les résultats présentés dans la Tableau 4 indiquent qu'il n'existe pas de différences significatives entre le nombre de panicules vides issues de l'association sorgho-Jatropha et celui des panicules vides provenant de la culture pure de sorgho $(\mathrm{P}>0,05)$, et ce, au cours des trois années d'étude. Le même résultat est observé pour le nombre de 
panicules à grains $(\mathrm{P}>0,05)$ entre les traitements et ce, au cours des trois années d'étude. Pour le poids de panicules, les traitements ne présentent aucune différence significative en première et en deuxième année d'étude. Par contre, en troisième année, on observe une différence significative entre le poids de panicules issues de l'association sorgho-Jatropha et celui du sorgho en culture pure. Le poids de panicules par hectare dans l'association Jatropha-sorgho est de 1499,54 $\mathrm{kg} \pm 191,71$ alors qu'il est de $1108,48 \mathrm{~kg} \pm$ 104,12 en culture pure de sorgho.

\section{Rendement grains du sorgho}

Pour le rendement grains de sorgho, les traitements en comparaison (Figures 1a, 1b et 1c) présentent des différences significatives la première année $(\mathrm{p}=0,002)$, la deuxième année $(\mathrm{p}=0,036)$ et la troisième année $(\mathrm{p}=0,010)$. Les rendements grains de sorgho sont plus élevés au niveau de l'association sorghoJatropha par rapport au rendement observé en culture pure de sorgho. La hausse de rendement du sorgho dans le système sorghoJatropha est de $29 \%$ à la première année, $30 \%$ à la deuxième année et $43 \%$ à la troisième année par rapport au rendement du sorgho en culture pure.

\section{Rendement paille du sorgho}

Les rendements paille du sorgho associé au Jatropha, présentent les mêmes tendances évolutives que les rendements grains pendant les trois années de l'étude (Figures 2a, 2b et 2c). Les résultats des analyses montrent que les parcelles du système sorgho-Jatropha produisent plus de biomasse aérienne que celles en culture pure de sorgho. En première année d'expérimentation, la quantité de biomasse du sorgho cultivé en association avec Jatropha est supérieure de $74 \%$ à celle du sorgho en culture pure. En deuxième et en troisième année, la biomasse du sorgho associée à la plantation de Jatropha représente respectivement $98 \%$ et $49 \%$ de celle obtenue avec le sorgho en culture pure.

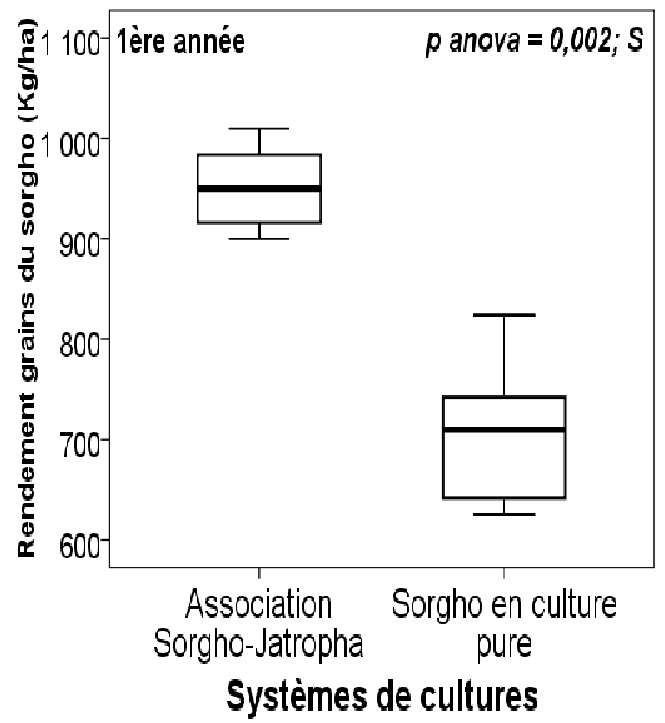




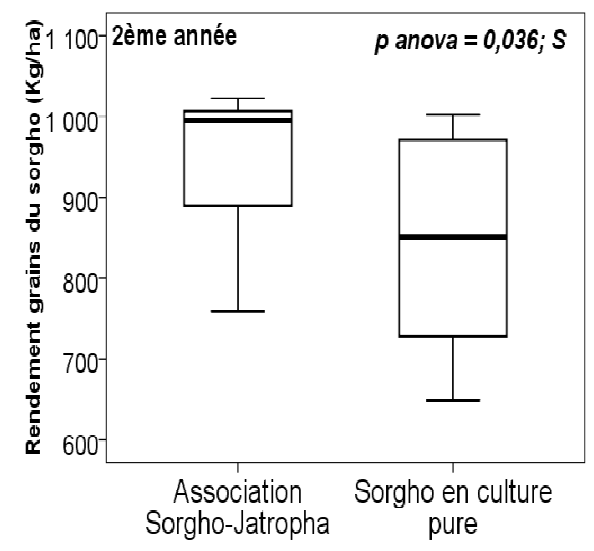

Systèmes de cultures

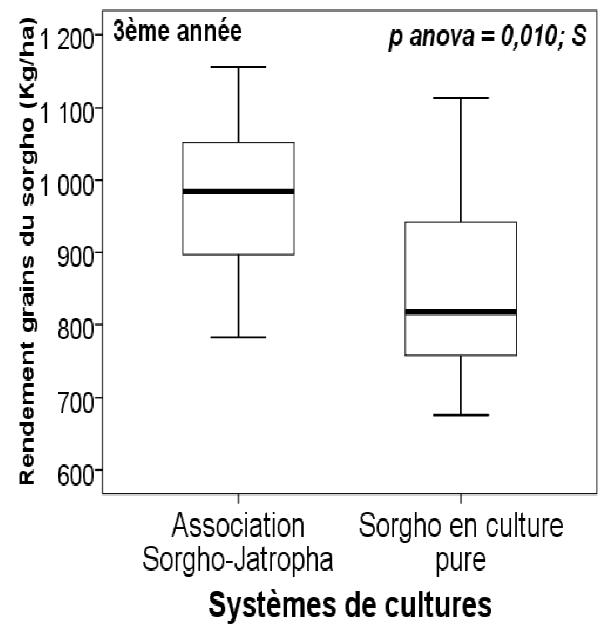

Figure 1: Evolution des rendements grains.

a) $1^{\text {ère }}$ année, b) $2^{\text {ème }}$ année, c) $3^{\text {ème }}$ année. Les barres représentent la médiane.

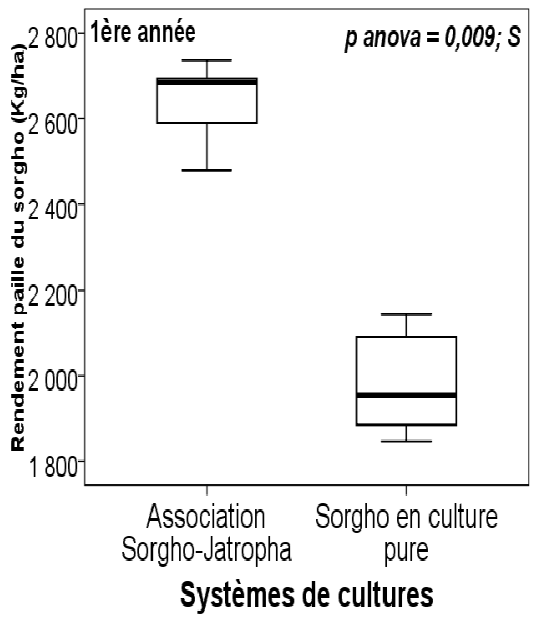




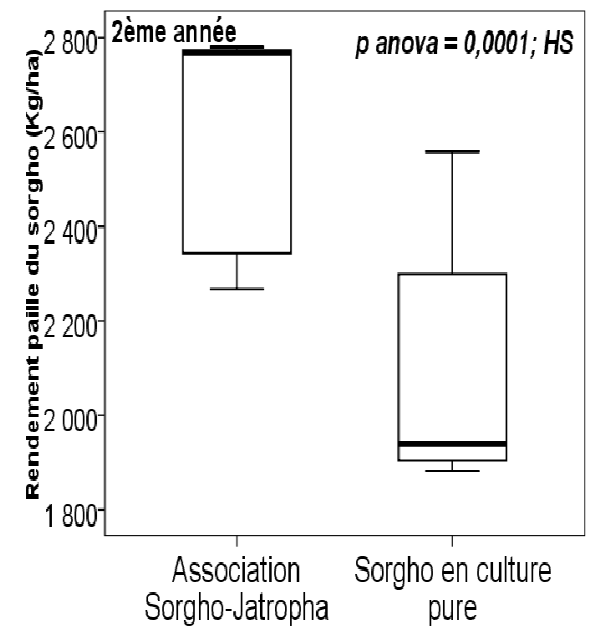

Systèmes de cultures

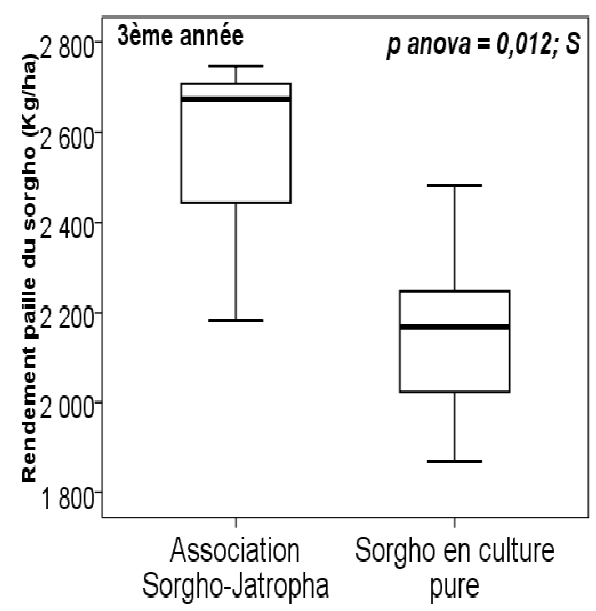

Systèmes de cultures

Figure 2: Evolution des rendements pailles.

a) $1^{\text {ère }}$ année, b) $2^{\text {ème }}$ année, c) $3^{\text {ème }}$ année. Les barres représentent la médiane.

Tableau 1: Pluviométrie des dix dernières années à Torokoro (2005-2014). DPARHASA COMOE, 2014

\begin{tabular}{lcccccccccc}
\hline Année & $\mathbf{2 0 0 5}$ & $\mathbf{2 0 0 6}$ & $\mathbf{2 0 0 7}$ & $\mathbf{2 0 0 8}$ & $\mathbf{2 0 0 9}$ & $\mathbf{2 0 1 0}$ & $\mathbf{2 0 1 1}$ & $\mathbf{2 0 1 2}$ & $\mathbf{2 0 1 3}$ & $\mathbf{2 0 1 4}$ \\
\hline Hauteur de pluie (mm) & 853,9 & 1238,9 & 1025 & 949,4 & 789,5 & 738,8 & 1073,6 & 1151,4 & 1058,09 & 1286,8 \\
Nombre de Jours (j) & 53 & 74 & 67 & 59 & 52 & 51 & 65 & 76 & 76 & 80 \\
\hline
\end{tabular}


Tableau 2: Evolution des paramètres chimiques du sol en fonction de la distance de prélèvement du sol.

\begin{tabular}{|c|c|c|c|c|c|c|c|c|c|c|}
\hline $\begin{array}{l}\text { Distance de } \\
\text { prélèvemen }\end{array}$ & & pHeau & pHkcl & $\begin{array}{c}\text { Carbone } \\
(\%)\end{array}$ & MO (\%) & $\mathbf{N}(\%)$ & $\mathrm{C} / \mathrm{N}$ & $\begin{array}{c}P_{-} \text {total } \\
\text { mg/kg sol }\end{array}$ & $\begin{array}{c}\text { K_total } \\
\text { mg/kg sol }\end{array}$ & $\begin{array}{c}P_{\text {P_assimilable }} \\
\text { mg/kg sol }\end{array}$ \\
\hline D0 & & $6,24 a \pm 0,27$ & $5,46 a \pm 0,24$ & $0,61 \mathrm{a} \pm 0,11$ & $0,97 a \pm 0,20$ & $0,05 a \pm 0,01$ & $13,39 \mathrm{a} \pm 3,01$ & $95,50 \mathrm{a} \pm 5,73$ & $767,63 a \pm 164,36$ & $6 \quad 1,91 \mathrm{a} \pm 0,53$ \\
\hline D1 & & $6,11 \mathrm{a} \pm 0,24$ & $5,26 a \pm 0,47$ & $0,44 \mathrm{~b} \pm 0,07$ & $0,80 b c \pm 0,12$ & $0,04 a \pm 0,01$ & $11,58 \mathrm{a} \pm 1,60$ & $88,42 \mathrm{ab} \pm 21,06$ & $772,78 a \pm 135,65$ & $1,86 a \pm 0,33$ \\
\hline D2 & & $6,04 a \pm 0,26$ & $5,16 a \pm 0,62$ & $0,44 \mathrm{~b} \pm 0,06$ & $0,72 \mathrm{c} \pm 0,12$ & $0,04 a \pm 0,01$ & $12,33 a \pm 1,86$ & $6 \quad 69,17 b \pm 8,49$ & $595,89 a \pm 91,05$ & $1,06 b \pm 0,20$ \\
\hline Témoin & & $6,20 \mathrm{a} \pm 0,58$ & $5,37 a \pm 0,66$ & $0,51 \mathrm{ab} \pm 0,09$ & $0,89 \mathrm{~b} \pm 0,22$ & $0,05 a \pm 0,01$ & $12,82 \mathrm{a} \pm 0,66$ & $6 \quad 82,47 \mathrm{ab} \pm 32,77$ & $793,61 \mathrm{a} \pm 29,75$ & $1,32 \mathrm{ab} \pm 0,76$ \\
\hline $\operatorname{Pr}>\mathrm{F}$ &, 531 & & & & & & & 0,012 & 0,117 & 0,008 \\
\hline Signification & $\mathrm{NS}$ & $\mathrm{Ns}$ & & $\bar{S}$ & $\mathrm{~S}$ & $\mathrm{JS}$ & NS & $\mathrm{S}$ & NS & $\mathrm{S}$ \\
\hline
\end{tabular}

Les moyennes dans chaque colonne affectées de la même lettre ne sont pas significativement différentes au seuil de probabilité 5\%. D0= sous houppier du Jatropha,

$\mathrm{D} 1=1 \mathrm{~m}$ du houppier, $\mathrm{D} 2=2 \mathrm{~m}$ du houppier. $\mathrm{pH}:$ Potentiel hydrogène $; \mathrm{KCl}:$ Chlorure de potassium $; \mathrm{C}:$ carbone $; \mathrm{MO}:$ matière organique $; \mathrm{N}:$ azote total $;$

$\mathrm{C} / \mathrm{N}$ : carbone /azote ; P_total : phosphore total ; K_total : potassium total ; P_ass : phosphore assimilable ; Pr $>\mathrm{F}$ : Probabilité observée ; S : Significatif ; NS : Non significatif.

Tableau 3: Croissance du sorgho dans le système sorgho-Jatropha à Torokoro.

\begin{tabular}{|c|c|c|c|c|}
\hline \multirow{2}{*}{$\begin{array}{c}\text { Année } \\
\text { d'expérimentation }\end{array}$} & \multirow{2}{*}{ Système de culture } & \multicolumn{3}{|c|}{ Hauteurs des plants de sorgho $(\mathrm{cm})$} \\
\hline & & 30 JAS & 60 JAS & $90 \mathrm{JAS}$ \\
\hline \multirow{4}{*}{$1^{\text {ère }}$ année } & Sorgho-Jatropha & $43,66 \mathrm{a} \pm 4,71$ & $127,56 \mathrm{a} \pm 22,37$ & $255,20 \mathrm{a} \pm 50,12$ \\
\hline & Sorgho pur & $33,40 \mathrm{~b} \pm 2,24$ & $114,44 \mathrm{a} \pm 15,91$ & $201,40 \mathrm{~b} \pm 6,69$ \\
\hline & $\operatorname{Pr}>\mathrm{F}$ & 0,014 & 0,316 & 0,045 \\
\hline & Signification & $\mathrm{S}$ & NS & $\mathrm{S}$ \\
\hline \multirow{4}{*}{$2^{\text {ème }}$ année } & Sorgho-Jatropha & $45,00 \mathrm{a} \pm 5,15$ & $122,28 \mathrm{a} \pm 21,06$ & 249,20 a $\pm 42,73$ \\
\hline & Sorgho pur & $37,60 \mathrm{~b} \pm 3,36$ & $125,80 \mathrm{a} \pm 10,89$ & $204,00 \mathrm{~b} \pm 8,15$ \\
\hline & $\operatorname{Pr}>\mathrm{F}$ & 0.027 & 0.748 & 0.049 \\
\hline & Signification & $\mathrm{S}$ & NS & $\mathrm{S}$ \\
\hline \multirow{4}{*}{$3^{\text {ème }}$ année } & Sorgho-Jatropha & $51,98 \mathrm{a} \pm 7,71$ & $178,40 \mathrm{a} \pm 21,11$ & 309,60 a $\pm 41,33$ \\
\hline & Sorgho pur & $46,42 \mathrm{a} \pm 5,23$ & $149,14 \mathrm{~b} \pm 6,22$ & $239,34 \mathrm{~b} \pm 11,15$ \\
\hline & $\operatorname{Pr}>\mathrm{F}$ & 0,219 & 0,018 & 0,024 \\
\hline & Signification & NS & $\mathrm{S}$ & $\mathrm{S}$ \\
\hline
\end{tabular}

Les chiffres portant les mêmes lettres ne sont pas significativement différents au seuil de probabilité 5\%. Pr>F Probabilité observée;

S: Significatif; NS : Non significatif. 
Tableau 4 : Nombre et Poids des panicules de sorgho dans le système sorgho-Jatropha à Torokoro.

\begin{tabular}{rlccc}
\hline Annéé & $\begin{array}{l}\text { Systèmes de } \\
\text { cultures }\end{array}$ & $\begin{array}{c}\text { Nbr Panicules } \\
\text { vides/ha }\end{array}$ & $\begin{array}{c}\text { Nbr Panicules } \\
\text { à grains/ha }\end{array}$ & $\begin{array}{c}\text { Poids panicules } \\
(\mathbf{k g} / \mathbf{h a})\end{array}$ \\
\hline \multirow{3}{*}{$1^{\text {ère }}$} & Sorgho-Jatropha & $4077,99 \mathrm{a} \pm 89,14$ & $73557,35 \mathrm{a} \pm 98,41$ & $1339,26 \mathrm{a} \pm 10,88$ \\
année & Sorgho pur & $4158,80 \mathrm{a} \pm 50,22$ & $72249,27 \mathrm{a} \pm 96,20$ & $1156,14 \mathrm{a} \pm 25,20$ \\
\cline { 2 - 5 } & Pr>F & 0,903 & 0,965 & 0,350 \\
& Signification & $\mathrm{NS}$ & $\mathrm{NS}$ & $\mathrm{NS}$ \\
\hline \multirow{2}{*}{$2^{\text {ème }}$} & Sorgho-Jatropha & $3685,40 \mathrm{a} \pm 92,11$ & $76133,06 \mathrm{a} \pm 97,32$ & $1425,26 \mathrm{a} \pm 18,44$ \\
année & Sorgho pur & $3863,28 \mathrm{a} \pm 27,43$ & $73280,23 \mathrm{a} \pm 68,54$ & $1309,35 \mathrm{a} \pm 14,28$ \\
\cline { 2 - 5 } & Pr>F & 0.876 & 0.338 & 0.353 \\
& Signification & $\mathrm{NS}$ & $\mathrm{NS}$ & $\mathrm{NS}$ \\
\hline \multirow{2}{*}{$3^{\text {ème }}$} & Sorgho-Jatropha & $3478,39 \mathrm{a} \pm 91,26$ & $79355,18 \mathrm{a} \pm 84,71$ & $1499,54 \mathrm{a} \pm 98,62$ \\
année & Sorgho pur & $3861,31 \mathrm{a} \pm 78,74$ & $75172,97 \mathrm{a} \pm 61,58$ & $1108,48 \mathrm{a} \pm 14,91$ \\
\cline { 2 - 5 } & Pr>F & 0,693 & 0,762 & 0,036 \\
& Signification & $\mathrm{NS}$ & $\mathrm{NS}$ & $\mathrm{S}$ \\
\hline
\end{tabular}

Les chiffres portant les mêmes lettres ne sont pas significativement différent au seuil de probabilité 5\%. Pr>F : Probabilité observée ; S : Significatif ; NS : Non significatif. Nbr : Nombre.

\section{DISCUSSION}

Influence des haies de Jatropha sur les propriétés chimiques du sol

Les $\mathrm{pH}$ obtenus compris entre 6,1 et 6,95 sont peu favorables à la pratique de la culture en les comparants aux normes de classification du Bureau National des Sols (BUNASOLS) du Burkina Faso. Cette acidité des sols n'est pas imputable à la présence de Jatropha, car les sols du site d'expérimentation sont de nature acide (Bado, 2002).L'on peut s'interroger sur le rôle tampon de Jatropha curcas L. sur le sol par l'apport de matière organique qu'il génère. Girard et al. (2011) ont montré que le pH de la rhizosphère peut varier de 1 à 2 unités par rapport au sol environnant. La production importante de biomasse par Jatropha qui retourne au sol conduit à des teneurs élevées de carbone au voisinage des pieds de Jatropha. Par ailleurs, les exsudats racinaires dont est issu le carbone sont, selon Soulama (2008), une source importante de matière organique, de carbone et d'azote pour le sol. L'augmentation de la teneur de l'azote avec celle du carbone est en accord avec les résultats de Legendre (2008) et Leye et al.
(2009) qui ont fait ressortir l'action des bactéries symbiotiques fixant l'azote au niveau des racines de Jatropha. Ces résultats confirment les précédents travaux de Bazongo (2011), qui a souligné que la litière de Jatropha se caractérise par de fortes teneurs en carbone et en matière organique et aussi par une teneur non négligeable en azote. Les valeurs $\mathrm{C} / \mathrm{N}$ des sols se situent entre10 et 15 . La valeur du rapport $\mathrm{C} / \mathrm{N}$ du sol augmente au fur et à mesure qu'on se rapproche de la haie de Jatropha. La minéralisation sous houppier est plus intense que celle hors houppier et pourrait s'expliquer par une activité biologique plus intense sous houppier. Le sorgho associé au Jatropha bénéficie des éléments minéraux issus de la minéralisation de la litière provenant de Jatropha. Les teneurs en phosphore total et en phosphore assimilable ont les plus fortes valeurs en se rapprochant de la haie de Jatropha est donc une source de phosphore et de potassium dans le sol bien que ces apports soient faibles (Latalpie, 2007; Legendre, 2008; Laviola et Dias., 2008). La présence de Jatropha semble favoriser la rétention du $\mathrm{P}$ et du $\mathrm{K}$ dans le sol. Ces résultats confirment ceux de Sanou 
(2010) qui a mis en évidence l'influence du houppier de Jatropha curcas L. sur la variation de la teneur en phosphore assimilable du sol.

\section{Effet des haies de Jatropha sur la croissance et le développement du sorgho}

L'étude montre que le sorgho issu du système sorgho-Jatropha croît plus vite que le sorgho cultivé en culture pure, et ce, pendant tout le cycle de la plante. Cela pourrait s'expliquer par le niveau de fertilité du sol. La matière organique, en se décomposant, est une source de nutriments pour les plants de sorgho dans l'association sorgho-Jatropha. Selon Soulama (2008) et Sene (2009), l'enfouissement dans le sol des feuilles de Jatropha curcas permet à des plants de sorgho d'obtenir une bonne croissance aérienne. Pour Soulama (2008) et Sanou (2010), la richesse $\mathrm{du}$ sol en azote, en carbone et en fractions fines sous couvert Jatropha a pu contribuer au développement des plants de sorgho. Les résultats de l'étude confirment ceux des travaux antérieurs de Bazongo (2011) qui a observé que Jatropha curcas L. a un effet favorable sur la croissance des plants de sorgho. Pour Danielo (2007), Jatropha peut être cultivé en association avec plusieurs cultures alimentaires sans compétition.

Effet des haies de Jatropha sur le poids des panicules du sorgho, le rendement grain et paille du sorgho

Le poids total des panicules dans le système sorgho-Jatropha est supérieur à celui des panicules de sorgho en culture pure. Ce résultat s'explique par la différence du niveau de fertilité du sol entre les deux systèmes. Le phosphore accélère la mise en graine, et le potassium stimule la constitution de la réserve nutritive (Bazongo, 2011; Yélémou et al., 2013). Jatropha favoriserait donc l'augmentation du poids total de panicule à l'hectare (Traoré et al., 2012). Les rendements grains enregistrés sont faibles par rapport au potentiel de la variété. Les rendements obtenus avec le sorgho associé au Jatropha au cours des trois années de l'étude sont plus importants par rapport à ceux observés avec le sorgho en culture pure. Ces sols sous la plantation de Jatropha sont plus fournis en matière organique et en éléments fertilisants comme l'azote, le phosphore et le potassium (Traoré et al., 2012). La matière organique en ameublissant le sol permet un bon enracinement $\mathrm{du}$ sorgho avec pour conséquence une bonne alimentation hydrique et minérale du sorgho qui est associé. Ceci pourrait expliquer les différences de rendement observées entre le sorgho associé au Jatropha et le sorgho en culture pure. L'action positive de Jatropha sur le rendement d'une culture du sorgho a été montrée par Bazongo (2011) qui a conclu à l'issue de ses travaux, que les meilleurs rendements sont obtenus avec du sorgho distant de $2 \mathrm{~m}$ de la haie de Jatropha. Des études similaires ont été menées avec le maïs par Tapsoba (2011) qui a conclu que les meilleurs rendements en maïs sont obtenus avec l'association maïs-Jatropha que le maïs en culture pure. Dans le système de culture sorgho-Jatropha, la biomasse aérienne produite par le sorgho est supérieure à celle obtenue en culture pure de sorgho. Le développement de la biomasse aérienne d'une culture est d'autant plus important que le sol est plus riche en éléments fertilisants comme l'azote, le phosphore et le potassium (Traoré et al., 2012; Yélémou et al., 2013). Ces éléments nutritifs, apportés par la litière et issus de Jatropha mais également par les exsudats racinaires, favorisent la croissance et le développement végétatif du sorgho associé. Ces résultats sont conformes aux conclusions des travaux de Bazongo (2011) qui a montré que les meilleurs rendements de biomasse sont obtenus pour des plants de sorgho situés à $1 \mathrm{~m}$ et à 2,5 $\mathrm{m}$ des plantations de Jatropha.

\section{Conclusion}

Afin d'évaluer l'influence de Jatropha curcas L. sur les propriétés chimiques du sol et sur la croissance et la productivité du sorgho, des essais ont été mis en place dans la 
zone Sud soudanienne du Burkina Faso. Les résultats montrent que la culture de Jatropha curcas L. améliore la teneur du sol en carbone, en matière organique et en azote (N). Jatropha curcas L. est une source non négligeable de phosphore et de potassium pour le sol et favorise la croissance du plant de sorgho qui lui est associé. Les travaux ont mis en évidence l'influence des haies de Jatropha curcas L. sur les composantes de rendement du sorgho. Jatropha contribue à améliorer la production de biomasse et le rendement du sorgho qui lui est associé. En termes de conseil technique, l'association culture céréalière-Jatropha peut être une solution à la résolution du manque de fourrage. Cette étude mérite d'être conduite sous d'autres zones pédoclimatiques en utilisant d'autres cultures associées comme les cultures maraîchères et les plantes à tubercules. L'utilisation des plantes à tubercules permettrait de comprendre les interactions racinaires de ces cultures associées avec Jatropha et d'étudier le phénomène de toxicité induite par Jatropha.

\section{CONFLIT D'INTERET}

Les auteurs déclarent ne pas avoir de conflit d'intérêt pour cet article.

\section{CONTRIBUTIONS DES AUTEURS}

PB a été l'investigateur principal; KT et OT ont supervisé, orienté l'étude et révisé le manuscrit; $\mathrm{BY}$ et $\mathrm{KBS}$ ont révisé le manuscrit. SK a le suivi les activités et la collecte les données. VH et BHN ont supervisé le travail et révisé le manuscrit

\section{REMERCIEMENTS}

Les auteurs traduisent également leur gratitude au Projet UA Jatropha de l'Institut de l'Environnement et de Recherche Agricole (Stations de Recherche de Kamboinsé et de Farako-Bâ) pour son soutien multiforme à la réalisation de cette étude.

\section{REFERENCES}

Adebowale KO, Adedire CO. 2006. Chemical composition and insecticidal properties of the underutilized Jatropha curcas seed oil. African Journal of Biotechnology, 5(10): $\quad 901-906 . \quad$ DOI: www.bioone.org/doi/abs/10.1673/031.00 9.6401

Bado BV. 2002. Rôle des légumineuses sur la fertilité des sols ferrugineux tropicaux des zones guinéenne et soudanienne du Burkina Faso. Thèse de Doctorat de troisième cycle, Université de Laval, Québec, p. 166.

Bazongo P. 2011. Introduction du Jatropha curcas L. dans les exploitations agricoles de la zone ouest du Burkina Faso : état des lieux et effet de la plante sur les propriétés des sols et des cultures associées. Mémoire de DEA en Sciences du Sol, IDR/Bobo-Dioulasso, p. 49.

Blind J, Dabat MH, Faugere G, Hanff E, Weisman N. 2008. Opportunités de développement des biocarburants au Burkina Faso. Ministère de l'Agriculture, de l'Hydraulique et des Ressources Halieutiques, Ouagadougou, Burkina Faso, p. 57.

BUNASOLS. 1987. Méthodes d'analyse physique et chimique des sols, eaux et plantes. Document technique $n^{\circ}$ 3. 48128.

Danielo O. 2007. Jatropha curcas: l'or vert du désert, p. 3.

Domergue M, Pirot R. 2008. Jatropha curcas L. Rapport de synthèse bibliographique, CIRAD, p. 118.

Diédhiou I. 2009. Impacts potentiels de l'introduction de Jatropha curcas L; dans un contexte de variabilité et changement climatiques impacts agricoles et environnementaux, intérêts économiques pour les ménages et communautés rurales. Atelier de clôture RIPIECSA, Cotonou, Bénin, 18-21 octobre. Université de Thiès, Sénégal.

DPARHASA COMOE. 2014. Synthèse pluviométrique de la zone de Mangodara. 
Rapport d'activité, Banfora, Burkina Faso, p.32.

Endelevu E. 2009. Jatropha Reality Check: A field assessment of the agronomic and economic viability of Jatropha and other oilseed crops in Kenya, p. 27.

Girard MC, Walter C, Rémy JC, Berthelin J, Morel JL. 2011. Sol et Environnement, (2ème édn). DUNOD : Paris ; 61-864.

Guillaume Z. 2009. Les impacts des biocarburants sur les zones humides ivoiriennes. Interview, Magazine radiophonique (Côte d'Ivoire/AMARC/ Convention Ramsar sur les zones humides/FAO), p. 3.

Henning KR. 2002. Utilisation des savoirs locaux sur Jatropha. Article, Note CA $\mathrm{n}^{\circ} \mathbf{4 2}$, p. 4.

Henning RK,Ramorafeno T. 2005. Le manuel Jatropha. Un guide pour l'exploitation intégrée de la plante Jatrophaà Madagascar. Etablie pour l'atelier de démonstration du Programme de Lutte Anti-Erosive (PLAE) à Morovoay (Madagascar), p. 20.

Latalpie R. 2007. La culture du poughère: une activité génératrice de revenus qui permet de faire face aux enjeux énergétiques du Mali. Cas du projet Garalo Bagani Yelen. Master, Université de Rennes1, p. 104.

Laviola BG, Dias LAS. 2008. Nutrient concentration in Jatropha curcas leaves and fruits and estimated extraction at harvest. Revista Brasileira de Ciência do Solo, 32: 1969-1975. DOI: http://dx.doi.org/10.1590/S010006832008000500018

Legendre B. 2008. Jatropha curcas (Tabanani). Note agronomique. Technologies for Human development, p. 8.

Leye EHM, Ndiaye M, Ndiaye F, Diallo B, Sarr AS, Diouf M, Diop T. 2009. Effet de la mycorhization sur la croissance et le développement de Jatropha curcas L. Revue des Energies Renouvelables, 12(2): 269278. www.cder.dz/download/Art12-2_10
Lottmann JH. 2008. Lessons to be learned for Tunisia from the cultivation and utilization of Jatropha curcas Linné (JCL) worldwide. Word-doc

Low T, Booth C. 2007. The weedy truth about Biofuels in Melbourne: the Invasive Species Council, 1-20.

Michaelson GJ, Ping CL, Mitchell GA. 1987. Correlation of Mehlich III, Bray-1 and ammonium acetate extractable $\mathrm{P}, \mathrm{K}, \mathrm{Ca}$ and $\mathrm{Mg}$ for Alaska agricultural soils. Communication in Soil Science and Plant Analysis, 18(9), USA. 1003-1015.

Ogbebor NO, Adekunle AT, Enobakhare DA. 2007. Inhibition of Colletotrichum gloeosporioides (Penz) Sac. Causal organism of rubber (Hevea brasiliensis Muell. Arg.) leaf spot using plant extracts. African Journal of Biotechnology, 6(3): 213-218. http://www.academicjournals.org/AJB

PANA. 2006. Programme d'Action National d'Adaptation à la variabilité et aux changements climatiques (PANA du BURKINA FASO), p. 76.

Paramathma M, Venkatachalam P, Sampathrajan A. 2007. Jatropha Improvement, Management and Production of Biodiesel. Centre of Excellence in Biofuels Agricultural Engineering. Book on the application of practical knowledge in energy crops College and Research Institute, Tamil $\mathrm{Nadu}$ Agricultural University, and Coimbatore 641003; 262.

Pellet JD, Pellet E. 2007. Jatropha curcas, le Meilleur des Biocarburants : Mode d'Emploi, Histoire et Avenir d'une Plante Extraordinaire. Favre : Lausanne; p1.

Sanou F. 2010. Productivité de Jatropha curcas L. et impact de la plante sur les propriétés chimiques du sol: cas de Bagré (Centre Est du Burkina Faso. Mémoire de fin de cycle, Université. Polytechnique de Bobo-Dioulasso, p. 55.

Soulama S. 2008. Influence du Jatropha curcas dans la séquestration du carbone et 
essai de compostage. Mémoire de fin d'études, SVT/UO, p. 56.

Sene M. 2009. Faisabilité de traitement des eaux usées domestiques par filtres de plantes d'espèces utilitaires: Jatropha curcas L. au Burkina Faso. Mémoire de fin d'études, 2iE, p. 55.

Tapsoba A. 2011. Réponses physiologiques des plantes vivrières cultivées sous plantation de Jatropha curcas L. cas du maïs, Zea mays L., dans la commune de Boni, en zone soudano sahélienne. Mémoire d'Ingénieur. IDR, p. 37.

Traoré M, Nacro HB, Tabo R, Nikièma A, Ousmane H. 2012. Potential for agronomical enhancement of millet yield via Jatropha curcas oilcake fertilizer amendment using placed application technique. Int. J. Biol. Chem. Sci., 6(2): 808-819. DOI http://dx.doi.org/10.4314/ijbcs.v6i2.23

Vedie H, Geffroy T. 2005. Lutte contre les nématodes à galles : Test de différents engrais verts nématicides laboratoire de nématologie du CBGP / IRD de Montpellier, France, p. 5.

Walkley A, Black R N. 1934. An examination of the method Dedtjareff for determining soil organic matter and to proposed modification of the chromic acid titration method. Soil Science, 37: 29-38.

Yélémou B, Yaméogo G, Barro A, Taonda SJ, Hien V. 2013. La production de sorgho dans un parc à Piliostigma reticulatum en zone nord soudanienne du Burkina Faso. Tropicultura, 31(3): 154-162. www.tropicultura.org/text/v31n3/154

Youl S. 2009. Dynamique et modélisation de la dynamique du carbone dans un agrosystème de savane de l'ouest du Burkina Faso. Doctorat Unique, Institut du Développement Rural/ Université Polytechnique de Bobo-Dioulasso, p. 186. 